\title{
Tcp Performance and Throughput Fairness Optimization in a Multi-Hop Pipeline Network
}

\author{
S.K. Subramaniam, I. Y. Panessai, R. A. Ramlee, R. Sujatha, N. Rajagopal
}

\begin{abstract}
Node starvation wireless sensor network (WSN) is a critical factor that affects the overall performance in a typical multi-hop linear network especially in an extensive scale network. The unfairness of sharing network resources with all source nodes in a multi-hop linear network amplifies the node starvation that often results in passive nodes in a network. This factor becomes critical with the increasing network density, aggressive data transfer, single destination node and inadequate data scheduling. This paper highlights the Delayed acknowledgement timeout for flat one-tier throughput critical application model (DAT-FTCAM) a mathematical fairness model that ensure maximum throughput fairness for pipeline network scenario. The DAT-FTCAM enables the users to calculate the maximum delayed acknowledgement timeout for transmission control protocol (TCP) proportional to the travel time or difference between a source and a destination node. The implementation of DAT-FTCAM technique with modified TCP parameters on NS2 has revealed a network fairness index of above 0.99 with optimum network performance in a scalable pipeline network. The DAT-FTCAM decreases data packet collision and eliminates passive nodes in a pipeline network with optimum throughput fairness.
\end{abstract}

Keywords : Delayed acknowledgement, Fairness index, Pipeline network, TCP.

\section{INTORDUCTION}

The deployment of a wireless sensor network (WSN) for remote condition monitoring in the oil and gas industry has a great demand over the years $[1,2]$. In general, the oil and gas remote monitoring consists of a communication backbone

Revised Manuscript Received on October 29, 2019

* Correspondence Author

S.K. Subramaniam*, Advance Sensors \& Embedded Controls System (ASECS), Centre for Telecommunication Research \& Innovation (CeTRI), Fakulti Kejuruteraan Elektronik dan Kejuruteraan Komputer, Universiti Teknikal Malaysia Melaka, Hang Tuah Jaya, 76100 Durian Tunggal, Melaka, Malaysia.Email: siva@utem.edu.my

I. Y. Panessai, department, Faculty of Art, Computing and Creative Industry, Universiti Pendidikan Sultan Idris, 35900 Tanjong Malim, Perak, Malaysia.. Email: ismailyusuf@fskik.upsi.edu.my

R. A. Ramlee, Electronic, Faculty of Electronics and Computer Engineering (FKEKK),Universiti Teknikal Malaysia Melaka (UTeM),Hang Tuah Jaya,76100 Durian Tunggal, Melaka, Malaysia. Email: ridza@utem.edu.my

R. Sujatha, department, 2Department of Embedded Technology (IoT and sensors specialization), School of Electronics and Communication Engineering, Vellore Institute of Technology, Vellore-632014, INDIA. Email: sujatha.r@vit.ac.in

N. Rajagopal, Department of Electronic \& Computer Engineering, College of Engineering, Design \& Physical Sciences, Brunel University London, UB8 3PH Uxbridge, United Kingdom.. Email: Rajagopal.Nilavalan@brunel.ac.uk that relays real-time data (measurements) from sensors located on critical pipeline points to a remote monitoring station where data analysis is performed [1, 3]. The transportion of a valuable data from the chain-like sensor arrays to the monitoring station, a routing algorithm predefines a communication path between sensor nodes to a destination node on a multi-hop linear topology. Thus, full deployment of WSN on pipeline network prompts to a series of performance related issues especially on resources sharing among source nodes [4-7]. Generally, a multi-hop linear WSN has severe performance degradation issues especially on network fairness using the Transmission Control Protocol (TCP) [8-10]. The network unfairness in TCP is a chain like an effect caused by node starvation, network density, aggressive data transfer, single destination node and inadequate data scheduling in a multi-hop linear topology. In a practical implementation, node starvation contributes towards passive nodes or nodes without data transmitting opportunity that degrades the network fairness as well as the overall network performance. The generated data packets from sensor nodes $\mathrm{Nn}$ and the respective data travel time factor is indicated in time $t$ to a centralised destination node ND as illustrated in Figure 1 [4].

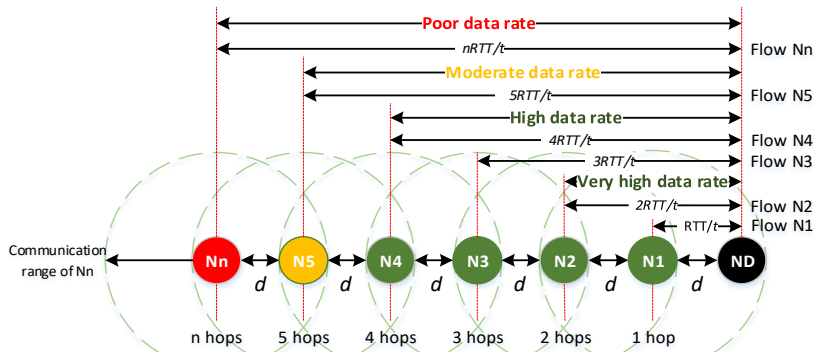

Fig. 1. The effect of data travel time in a typical pipeline network (linear topology)

In a real scale implementation, the travel time factor from data packet generation (source node level) to reach a destination node is subjected to the distance as shown in Figure 1 that effects the network fairness at time $t+1$. In such an architecture, improper data transfer rate and aggressive data transfer effects will amplify at time $t+2$ that results in source node starvation commonly among nodes located further away from the destination node [9-13]. It is a crucial issue in a multi-hop linear topology that leads to data packet loss and it's a waste of network resources.

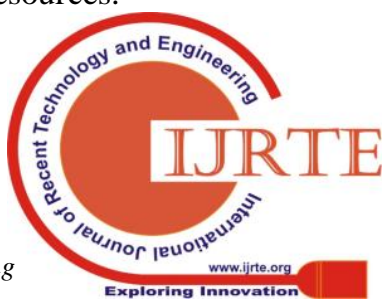




\section{Tcp Performance and Throughput Fairness Optimization in A Multi-Hop Pipeline Network}

\section{TCP AND RELATED WORKS ON NETWORK FAIRNESS}

In many IEEE 802.11 wireless network-based application, TCP is a popular protocol where is regarded as a high-performance protocol with congestion collapse avoidance can be achieved [14-16]. The fundamental operation of a TCP is to organise the data packet sending rate at an optimum state in a WSN. The advertised window is affiliated to the destination node while the congestion window is designed to overseas the utilisation of bandwidth for data packet transmission at the source node. In the recent of TCP version, multiple algorithms are incorporate as stated in (RFC 5681) for congestion avoidance, slow-start, fast retransmit, and fast recovery $[14,15]$. The Round Trip Time (RTT) as stated in RFC 2988 in TCP is known as one complete cycle time to send a data packet from a source node to a destination node with an acknowledgement packet returned to the respective sender [17]. An estimated RTT value used for the retransmission timeout (RTO) on a conventional TCP mechanism. In recent years, researchers have been working to enhancing TCP performance especially in congestion handling, handling of packet loss, decreasing errors etc. $[12,18]$. Therefore, several types of TCP algorithms are commonly available for users as required by the application.

The research on TCP has mainly focused on new protocols, techniques, applications and devices for achieving optimal fairness outcomes. The authors in [19] have suggested TCP-Optimal Queue Selection a dual queue technique with an optimal queue selection mechanism. In this technique, two independent queue mechanism is designed to hold the data packets in one queue while the acknowledgement packets on the second queue. The ideal queue size is defined by priority or probability to achieve better fairness in a network. In the priority scheduling, the acknowledgement packet is being priorities. While in the probability scheduling, the selection of queue referring to ideal queue size and queuing delay as for an acknowledgement packet is derived. The author in [12] designed Adaptive Delay Window on TCP (TCP-ADW) to reduce generated acknowledgement packets that are an accomplishment with an ideal dynamic delay window. The delay window designed within the cycle time that prevents the transmission timeout at the sender. This condition becomes void if the retransmission timer expires or the recipient enlarges the delay window based on the data rate.

The Adaptive Delayed ACK Mechanism (ADAM) was designed by $[10,20]$ to accurately adjust TCP parameters to achieve higher throughput fairness as well as to reduce frame collisions. The author in $[10,20]$ then enhanced ADAM to $\mathrm{ADAM}+$ to further improve throughput fairness in a network by eliminating the request to send (RTS) and the clear to send (CTS) packets. Another technique from the same author is two sender nodes in the same communication range. ADAM-snd has a probability of bypassing its intermediate node that can reduce the network RTT value. Both ADAM+ and ADAM-snd uses the fundamental concept and ADAM in shorter node distance (ADAM-snd) designed for

configuration of ADAM. The author in [21] designed the delayed cumulative acknowledgement (TCP-DCA) a technique with variable delay window size that changes to the generation TCP acknowledgement packet based on hop counts. Hop count or path length metrics that refers to the distance is used to control the delay window size for acknowledgement packets generated to the source nodes. The cooperation between channel access control along with TCP Rate Adaptation (CATRA) [22, 23] is designed to regulate an ideal Contention Window $(\mathrm{CW})$ size that is used to the control station contention. With the modified CW size helps to achieve better per-station fairness along with the channel utilisation information relayed to the transport layer that adjusts the TCP sending rate to achieve per-flow fairness. The author in [15] designed TCP-Vegas-W a technique to decrease aggressive window size for a multi-hop network. This technique helps to operate the congestion window at an appropriate level. Another technique called Monitoring Delayed Acknowledgment (TCP-MDA) introduced by [12] that interact with both TCP as well as MAC layer to minimise the acknowledgement packets through channel condition monitoring.

\section{DELAYED ACKNOWLEDGEMENT TIMEOUT MODEL FOR THROUGHPUT FAIRNESS CRITICAL APPLICATION}

This paper proposes the delayed acknowledgement timeout for flat one-tier throughput critical application model (DAT-FTCAM) designed for a TCP agent (recipient) with one receiver node in a multi-hop linear topology. Generally, the normalised throughput fairness index of 1.0 $(100 \%)$ is the desired value among source nodes in a multi-hop linear topology. This is often related to one receiver node with multiple source nodes and incrementing distance (meters) in linear.

In a wireless network, throughput is defined as the received data (total data packets) at a recipient in per unit time that is measured in bits/sec. In a wireless network, throughput is a critical parameter that represents the network capacity in terms of data transfer with the available resources. The average throughput (Kbps) in a network is as shown in equation $(1)[4,10]$ where PS represents packet size (defined in the simulation setup), RPi represents total received data packets for nn number of source nodes and tEnd- $\mathrm{tStart}$ represents the $\Delta$ Duration $\mathrm{t}$ within the simulation duration.

Throughput $=\frac{\sum_{i=1}^{n n}\left(\left(P S \times 8 \times R P_{i}\right) /\left(t_{\text {End }}-t_{\text {start }}\right)\right.}{n n}$

The throughput fairness index in a network is calculated from individual throughput from source nodes as shown in Jain's equation (2) [4, 10, 24] Where ni represents the normalised throughput (Kbps) for $\mathrm{n}$ flows with $\mathrm{N}$ represents the number of nodes.

Fairness index $=\frac{\left(\sum_{i=0}^{n} n_{i}\right)^{2}}{N \sum_{i=0}^{n} n_{i}^{2}}$ 
The desirable throughput fairness index of 1.0 indicates a network has $100 \%$ normalised throughput fairness in the network. The proposed technique in this paper deploys; (1) hop count (total travel cost in a network) between a source to destination node.

(2) to control and decrease packet collision in a multi-hop linear topology.

\section{A. Data travel cost in a network and the delayed acknowledgement mechanism}

The DAT-FTCAM considers the hop count (based on RTT) in time (t) among respective source nodes in a dedicated path (either odd path or even path whichever has a higher hop count) from a destination node. Generally, the hop is a unit count that is needed for a source node to transfer the data to its neighbouring node or beyond that is subjected to communication range and node separation from one another in the network. Generally, the hop count is calculated based on node distribution (meter) and hops required to move a data to a destination node in a network. Figure 2 represents a pipeline network with On/En source nodes and ND as the destination node [4]. (s) can send paper in the given email address of the journal. There are two email address. It is compulsory to send paper in both email address.

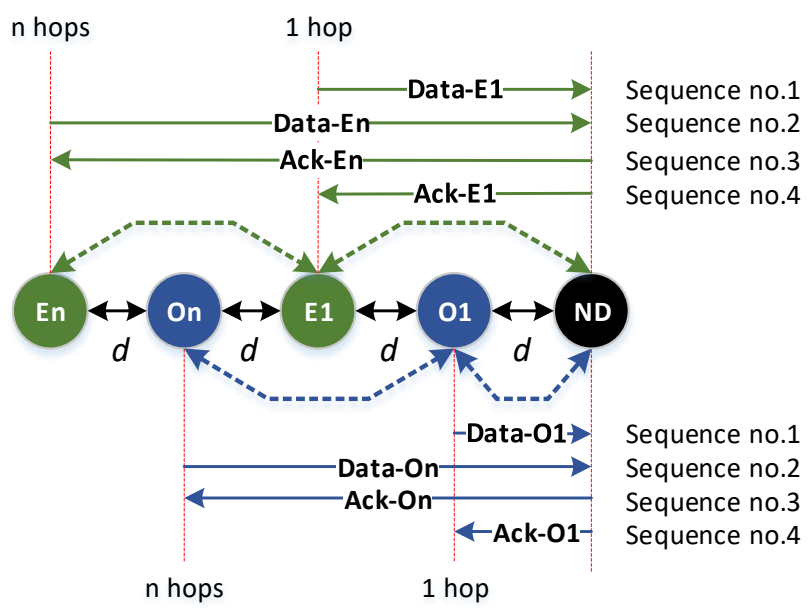

Fig. 2. A pipeline network with DAT-FTCAM

Figure 2 shows all nodes in a network uniformly distributed at $\mathrm{d}$ in meters with a maximum communication range is $2 \mathrm{~d}$ in meters. This setup enables a node to bypass a neighbouring node to transfer the data packets forward. Therefore, a minimum number of hop count (Hcmin) and a maximum number of hop count (Hcmax) for Figure 3 or similar network can be calculated from equation (3) and (4) respectively [4]. The value in Di represents the separation between a source to a destination node, $d$ represents the distribution of nodes (in meter) and $\mathrm{R}$ represents the maximum communication range (in meter).

$H c_{\min }=\left[\frac{D_{i}}{(R / d) \times d}\right]$
$H c_{\max }=\left[\frac{D_{i}}{d}\right]$

In the DAT-FTCAM, the value of RTT is multiplied with the total hop count to calculate an optimal delayed acknowledgement timeout for a set of source nodes based on the distance in a network. The total hop count and the factor of the transition period (n) in-between received data packets and generation of acknowledgement packets is an important parameter in determining the delayed acknowledgement timeout for respective source nodes as in equation (5-6). Equation (5) indicates the value of $\mathrm{n}$ can be determined by the number of interleaving path (Referring to Figure 2 the $n$ was set to one) in parallel to the calculated path.

The concept of transition time/phase was introduced in DAT-FTCAM to give room for any outstanding data packets on queue to be delivered to the destination node. The equation in $(5-6)$ can be used to calculate the needed delayed acknowledgement timeout for respective source nodes to achieve optimal throughput fairness index in a certain network. Where Fair cycle time $(\mathrm{t})$ represents a cycle time needed for nn number of source nodes and Hi represents the hop count for nn number of source nodes.

Fair cycle time $\left.(t)=\left(C \sum_{i=1}^{n n}\left(H_{i}\right)\right)+n\right) \times R T T$

The delayed acknowledgement timeout can be derived from equation (6) from the increasing sequence of source nodes arranged in linear as shown in Figure 2 or similar network to achieve optimal throughput fairness index. Where DACKnn represents the value used by the delayed acknowledgement timeout mechanism for nn number of source nodes in a linear arrangement (odd and even) and RTTnn is the respective source node RTT value referring to the sequence of location in a linear arrangement.

$D A C K_{n n}=$ Fair cycle time $(t)-R T T_{n n}$

With the proposed DAT-FTCAM, optimal throughput fairness index were achieved with the data transmission chronology by source nodes are in control with the delay acknowledgement timeout for the entire duration. Data packet collision is normal in a wireless system, particularly in a network with high data rate. Generally, packet collision results in unexpected data packet dropped that reduces the throughput fairness index as well as the waste of network resources. To retain a throughput fairness index of 1.0 among source nodes and to minimize the packet collision, the advertised window (awnd) buffer was set to one. The advertised window can avoid overflow (from source nodes) of network resources at a destination node. On the other end, a congestion window (cwnd) can avoid over sending data packets (from source nodes) than the permitted allocation. The congestion window is predefined and controlled by a TCP congestion control algorithm during active network period. The mathematical formulation in DAT-FTCAM is best implemented with a DI-LSR routing algorithm [4] for a series of source nodes in a pipeline network. The DI-LSR is a routing algorithm with low

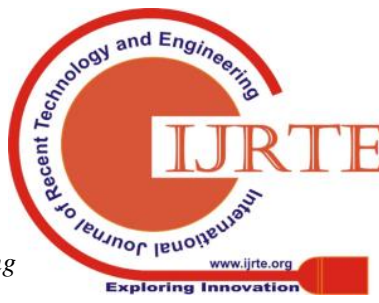


routing overhead and routes data in a predefined dual interleaving path between a sender and a destination node.

\section{B. Data and acknowledgement packet flow with DAT-FTFCAM in a pipeline network}

The data packet sending and receiving acknowledgement packet sequence with a transition waiting period in DAT-FTCAM with six source nodes (odd path: O1 to On, Figure 3, all source nodes $(\mathrm{O} 1 / \mathrm{E} 1 / \mathrm{O} 2 / \mathrm{E} 2 / \mathrm{On} / \mathrm{En})$ initiates the first data packet (Data-O1/Data-E1/Data-O2/Data-E2/Data-On/Data-En) transmission at time $t+1$ to the destination node (ND) at the start of each 1 Sum $\mathrm{H}$ cycle $(\mathrm{t})$ or fair cycle time $(\mathrm{t})$. The generated data packets (Data-O1/Data-E1) from source nodes $(\mathrm{O} 1 / \mathrm{E} 1)$ requires one hop to the designated destination node (ND) in sequence no. 1 at a duration of one RTT where else for the generated data packets (Data-O2/Data-E2) from source nodes (O2/E2) requires two hops through the intermediate node $(\mathrm{O} 1 / \mathrm{E} 1)$ to reach the destination node (ND) in sequence no. 2 with two RTT value. The generated data packets (Data-On/Data-En) from source nodes (On/En) requires three hops through the intermediate node $(\mathrm{O} 2 / \mathrm{E} 2$ and $\mathrm{O} 1 / \mathrm{E} 1)$ to reach the destination node (ND) in sequence no. 3 with three RTT value. Based on the mathematical formulation of DAT-FTCAM, the first acknowledgement packets (Ack-On/Ack-En) at sequence no. 4 will be generated at the destination node (ND) with three hops required through the intermediate node $(\mathrm{O} 1 / \mathrm{E} 1$ and $\mathrm{O} 2 / \mathrm{E} 2)$ to reach the respective source nodes $(\mathrm{On} / \mathrm{En})$ at the edge of seven RTT value. In the second acknowledgement packets at sequence no. 5 (Ack-O2/Ack-E2) from the destination node (ND) requires two hops to reach the respective source nodes $(\mathrm{O} 2 / \mathrm{E} 2)$ at the edge of seven RTT value. For the third and final acknowledgement packets at sequence no. 6 (Ack-O1/Ack-E1) from the destination node (ND) requires only one hop to reach the respective source nodes $(\mathrm{O} 1 / \mathrm{E} 1)$ at the edge of seven RTT value.

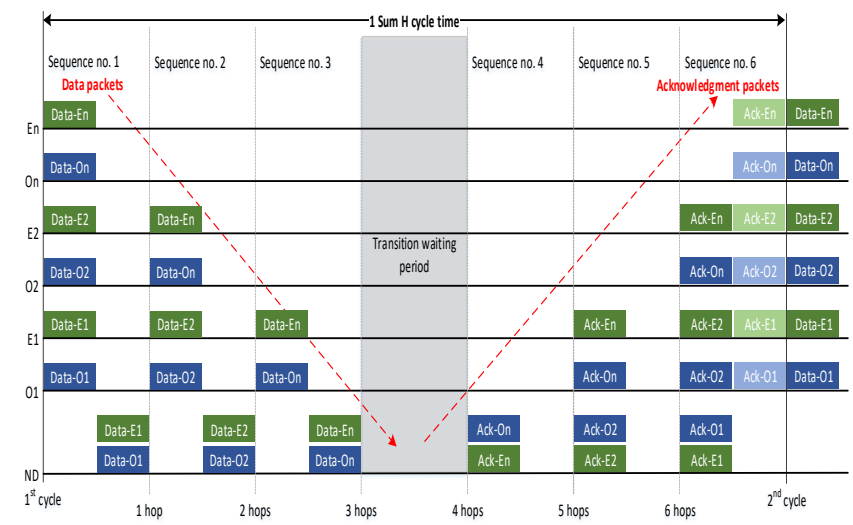

Fig. 3. Timing diagram with the implementation of DAT-FTCAM

\section{SIMULATION AND EVALUATION}

This section presents all the simulation results obtained from Network Simulator 2 (Version 2.35) [25] with the manipulated variable on a number of source nodes arranged even path E1 to En) is as shown in Figure 3 [4]. Referring to

in a linear topology. The simulation environment was re-created to match a real-life application (stationarily placed in the uniform distance) with two random function which is for data packet start time $(0.0-0.5$ seconds $)$ and non-sequence chronology of a source nodes. The simulation setting and parameters for DAT-FTCAM, AODV+ and DSDV+ is as described in Table-I.

\section{Table-I: NS2 simulation parameters}

\begin{tabular}{|c|c|}
\hline Parameters & Value \\
\hline $\begin{array}{l}\text { Channel type/MAC type/Radio } \\
\text { propagation model }\end{array}$ & Wireless channel/802.11/Two ray ground \\
\hline Interface queue type & DropTail/PriQueue \\
\hline Source nodes & $\begin{array}{l}12-120 \text { (increased by } 12 \text { nodes each } \\
\text { variation) }\end{array}$ \\
\hline Max packet in ifq & $\begin{array}{l}100 \text { (pkts) AODV+, DSDV+/50 (pkts) } \\
\text { DAT-FTCAM }\end{array}$ \\
\hline Agent type/Traffic type & TCP - DelAck/Constant bit rate (CBR) \\
\hline RTS/CTS & Disabled in all conditions \\
\hline $\begin{array}{l}\text { Packet size/ Simulation duration/ } \\
\text { RX Thresh/CS Thresh }\end{array}$ & $\begin{array}{l}125 \text { meters } / 250 \text { meters } / 512 \text { bytes } / 500 \\
\text { seconds }\end{array}$ \\
\hline
\end{tabular}

The simulated results are from an average of five cycle runs with a selection of seed values between 1-15. The results obtained for DAT-FTCAM incorporated with DI-LSR were compared with AODV+ (reactive protocol) and DSDV+ (proactive protocol) using the fairness mechanism proposed in [7] on the following metrics:

\section{A. Throughput fairness index}

The results on throughput fairness index shown in Figure 4 indicates that DAT-FTCAM has normalised fairness of above $99 \%$ among all source nodes as compared to AODV+ and DSDV+ in the network.

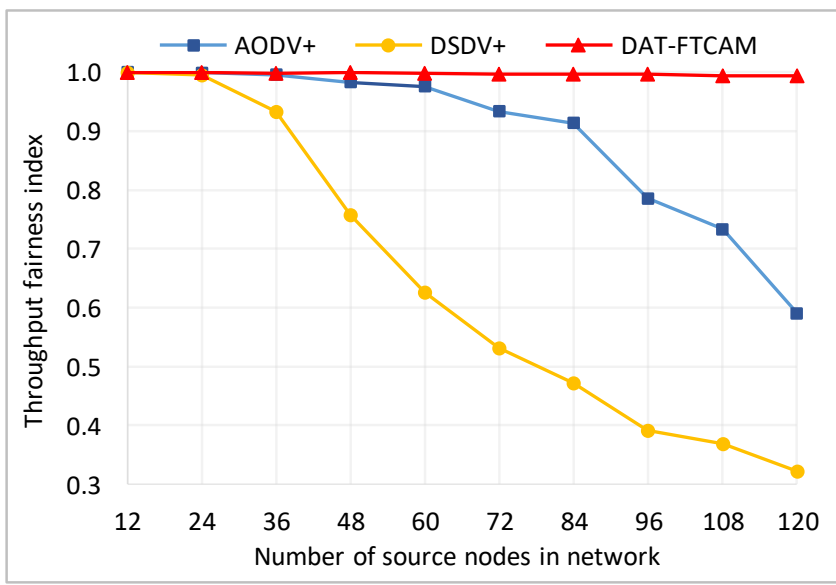

Fig. 4. Graph on throughput fairness index against the number of source nodes

A network with DAT-FTCAM has achieved a throughput fairness index above $0.99 / 1.0$ from a network of 12 nodes to a network of 120 nodes. While for both AODV+ and DSDV+ shown a similar degrading pattern below 0.733 and 0.3687 respectively in the same network setup of a network of 12 nodes to a network of 120 nodes. Appropriately calculating the delayed acknowledgement timeout ensure a timely slot for every source node in DAT-FTCAM instead of 
aggressive and concurrent data transmission. The time slot and reducing the advertised window in DAT-FTCAM also eliminates the number of passive nodes in a network. The results have indicated that with the implementation of DAT-FTCAM in a pipeline network ensures a balanced and equal share in network allocation with optimal throughput fairness index.

The DAT-FTCAM will be a feasible choice to be implemented on a pipeline network where a fair share of network resources is critical.

\section{B. Received data packet variation}

The received data packet variation as shown in Figure 5 is the other parameter measure network fairness, especially in a pipeline network. The results were interpreted in percentage rather than the number of the received data packet as a normalised value for better comparison. The results indicated the received data packet variation in percentage for DAT-FTCAM is relatively lower by half as compared with AODV+ and DSDV+ from a network of 12 nodes to a network of 120 nodes. For compared techniques, the effect on received data packet variation is proportional to the network density.

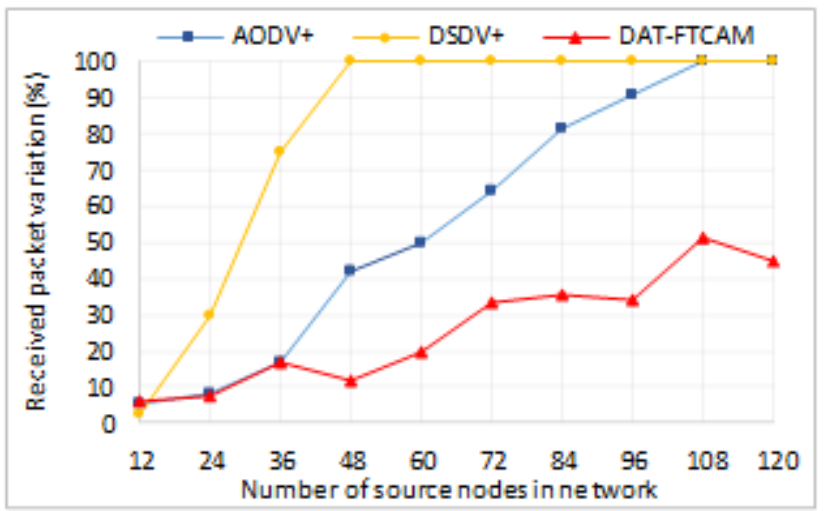

Fig. 5. Graph on received data packet variation against the number of source nodes

\section{Throughput}

A network that can achieve higher throughput within the available resources is always a desirable performance metrics in a WSN. The throughput results shown in Figure 6 indicated that a network with DAT-FTCAM outperforms AODV+ and DSDV+ from a network of 12 nodes to a network of 120 nodes.

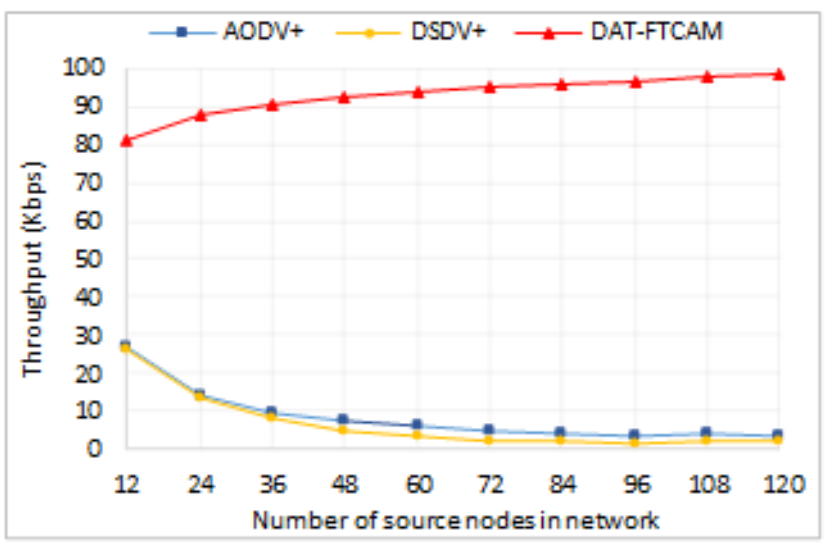

Fig. 6. Graph on throughput (Kbps) against the number of source nodes

The AODV+ and DSDV+ have a similar throughput curve pattern below $30 \mathrm{Kbps}$ while DAT-FTCAM has a significant difference of $54 \mathrm{Kbps}$ at 12 nodes to $96 \mathrm{Kbps}$ at 120 nodes. The time slot for source nodes in DAT-FTCAM established a non-aggressive data transfer and allocated equal data transmission opportunity for all source nodes in a network. A network with DAT-FTCAM enables higher data packet transfer by retaining optimal throughput fairness index as shown in Figure 5 which makes it a desirable choice for a pipeline network.

\section{Delivery ratio}

The results on the delivery ratio as shown in Figure 7 indicated a network can achieve a constant rate of above $99 \%$ from a network of 12 nodes to a network of 120 nodes with the implementation of DAT-FTCAM as compared with AODV+ and DSDV+. Generally, AODV+ and DSDV+ have a degrading effect on the delivery ratio rate up to $63 \%$ and $33 \%$ respectively. The time slot for source nodes in DAT-FTCAM established a non-aggressive data transfer and allocated equal data transmission opportunity for all source nodes in a network to ensure higher data transfer rate. A network with higher delivery ratio indicates the optimum use of available network resources that is measured in throughput. The packet collision can be minimised with DAT-FTCAM since every source node data packet were acknowledged at a different time interval with a minimum number of the advertised window. Hence, the implementation of DAT-FTCAM has also indicated a more reliable and scalable network in a pipeline network. 


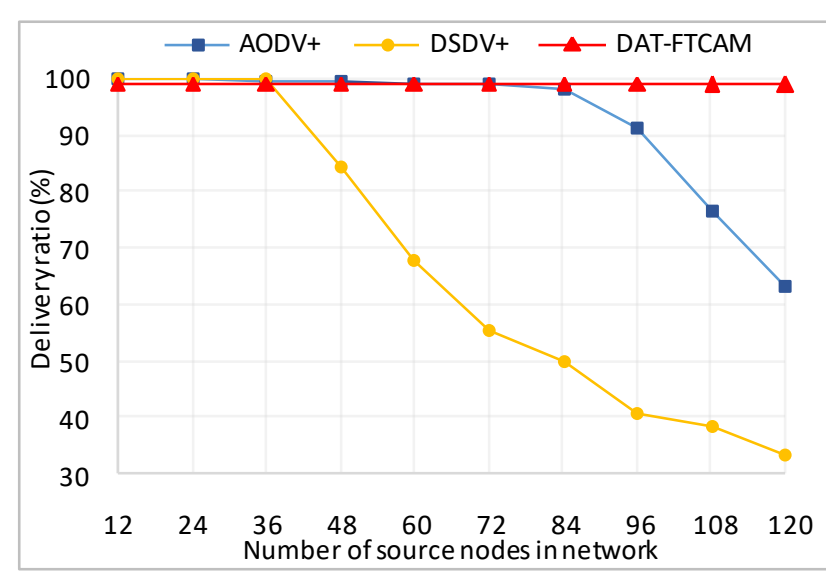

Fig. 7. Graph on received packet variation against the number of source nodes

\section{CONCLUSION}

This paper presents the outcome of the DAT-FTCAM associated with a TCP agent which was designed to achieve optimal throughput fairness index for a pipeline network. The node location and separation between a sender and a recipient in a linear arrangement is a crucial factor to network imbalance or unfairness that contributes to note starvation. Node starvation significantly contributes to the overall network performance in a WSN. Implementing the DAT-FTCAM through a mathematical formulation to determine an ideal delayed acknowledgement timeout for source nodes and lowering the advertised window size enhances the overall network performance in a pipeline network. Furthermore, this technique improves the resources sharing in a network and reduce the data packet collisions in a network.

The proposed DAT-FTCAM is feasible for deployment on a pipeline network with DI-LSR to achieve a throughput fairness index of above 0.99 (99\%) with zero passive nodes which were verified through NS2. The simulation results also show significant increases in throughput (Kbps) by $97 \%$ as compared with other fairness models. The unique formulation in DAT-FTCAM retains the throughput fairness index along with throughput for a scalable pipeline network.

\section{ACKNOWLEDGMENT}

This research is part of a grant entitled Analysis and implementation of an IoT based remote monitoring of oil and gas pipelines, grant no. PJP/2018/FKEKK (5B)/S01618 which was funded by Universiti Teknikal Malaysia Melaka. The authors would like to thank Ministry of Higher Education Malaysia for their financial support and Brunel University London for their lab facilities.

\section{REFERENCES}

1. S. Ali, S.B. Qaisar, H. Saeed, M.F. Khan, M. Naeem and A. Anpalagan, "Network Challenges for Cyber Physical Systems with Tiny Wireless Devices: A Case Study on Reliable Pipeline Condition Monitoring," Sensors, vol. 15, pp. 7172-7205, 2015.

2. H. Devold, Oil and gas production handbook: an introduction to oil and gas production, Lulu Press, 2013.

3. W.Z. Khan, M.Y. Aalsalem, M.K. Khan, M.S. Hossain and M. Atiquzzaman, "A reliable Internet of Things based architecture for oil and gas industry," in Advanced Communication Technology (ICACT), 2017 19th International Conference on, pp. 705-710, 2017.

4. S.K. Subramaniam, "Novel Design of Reliable Static Routing Algorithm for Multi-Hop Linear Networks", Ph.D. dissertation, Department of Electronic \& Computer Engineering, Brunel University London, 2017.

5. S. Datta and S. Sarkar, "A review on different pipeline fault detection methods," J Loss Prev Process Ind, vol. 41, pp. 97-106, 2016.

6. A. Jamatia, K. Chakma, N. Kar, D. Rudrapal and S. Debbarmai, "Performance Analysis of Hierarchical and Flat Network Routing Protocols in Wireless Sensor Network Using Ns-2," International Journal of Modeling and Optimization, vol. 5, pp. 40-43, 2015.

7. S. Varshney, C. Kumar and A. Swaroop, "Linear sensor networks: Applications, issues and major research trends," in Computing, Communication \& Automation (ICCCA), 2015 International Conference on, pp. 446-451, 2015.

8. I. Jawhar, N. Mohamed and D.P. Agrawal, "Linear wireless sensor networks: Classification and applications," Journal of Network and Computer Applications, vol. 34, pp. 1671-1682, 2011.

9. V. Chawla, "Performance Analysis of TCP-IA for Multi-Hop Wireless Networks," International Journal of Emerging Trends in Engineering and Development, vol. Vol. 2, pp. 521 - 526, May 2013.

10. T. Hou, C. Hsu and C. Wu, "A delay-based transport layer mechanism for fair TCP throughput over 802.11 multihop wireless mesh networks," International Journal of Communication Systems, vol. 24, pp. 1015-1032, 2011.

11. A.M. Al-Jubari, M. Othman, B.M. Ali and Hamid, Nor Asilah Wati Abdul, "TCP performance in multi-hop wireless ad hoc networks: challenges and solution," EURASIP Journal on Wireless Communications and Networking, vol. 2011, pp. 1-25, 2011.

12. A.M. Al-Jubari, M. Othman, B.M. Ali and Hamid, Nor Asilah Wat sAbdul, "An adaptive delayed acknowledgment strategy to improve TCP performance in multi-hop wireless networks," Wireless Personal Communications, vol. 69, pp. 307-333, 2013

13. Y. Chan, C. Lin, C. Chan and C. Ho, "CODE TCP: a competitive delay-based TCP," Comput.Commun., vol. 33, pp. 1013-1029, 2010.

14. V. Jacobson, "Congestion avoidance and control," ACM SIGCOMM Computer Communication Review, vol. 25, pp. 157-187, 1995.

15. M. Allman, V. Paxson and E. Blanton, "TCP Congestion Control," Tech. Rep. Request for Comments: 5681, 2009.

16. V. Patil and P. Chougule, "Survey of Different TCP Protocols," International Journal of Engineering Technology, Management and Applied Sciences, vol. 3, pp. 37-42, 2015.

17. M. Sargent, M. Allman and V. Paxson, "Computing TCP's Retransmission Timer," Computing, 2011.

18. H. Shi, R.V. Prasad, E. Onur and I.G. Niemegeers, "Fairness in wireless networks: Issues, measures and challenges," Communications Surveys \& Tutorials, IEEE, vol. 16, pp. 5-24, 2014.

19. S.S. Priya and K. Murugan, "Enhancing TCP Fairness in Wireless Networks using Dual Queue Approach with Optimal Queue Selection," Wireless Personal Communications, vol. 83, pp. 1359-1372, 2015.

20. C. Hsu and T. Hou, "Achieving fair throughput for tcp flows in multi-hop wireless backhaul networks," in Communications (ICC), 2010 IEEE International Conference on, pp. 1-5, 2010.

21. B. Chen, I. Marsic, H. Shao and R. Miller, "Improved delayed ack for tcp over multi-hop wireless networks," in IEEE Wireless Communications and Networking Conference (WCNC 2009), pp. 1-5, 2009.

22. P.T. Giang and P.M. Vi, "Cross layer design to enhance TCP performance in multi-hop ad hoc networks," in Advanced Technologies for Communications (ATC), 2013 International Conference on, pp. 642-647, 2013.

23. P.T. Giang and K. Nakagawa, "Achieving fairness over 802.11 multihop wireless ad hoc networks," IEICE Trans.Commun., vol. 92, pp. 2628-2637, 2009

24. R. Jain, D. Chiu and W.R. Hawe, A quantitative measure of fairness and discrimination for resource allocation in shared computer system, Eastern Research Laboratory, Digital Equipment Corporation Hudson, MA, 1984.

25. T. Issariyakul and E. Hossain, Introduction to network simulator NS2, Springer Science \& Business Media, 2011. 


\section{AUTHORS PROFILE}

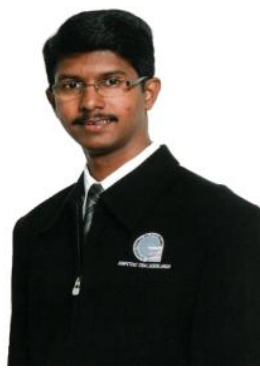

Dr Siva Kumar Subramaniam is a Senio Lecturer at Universiti Teknikal Malaysia Melaka, where he has been since 2006. He completed his Diploma in Electronics Engineering from Politeknik Ungku Omar in 2002. Siva Kumar Subramaniam than received a B.Eng in Electronics Engineering (Industrial Electronics) from Kolej Universiti Teknikal Kebangsaan Malaysia in 2006 and M.Sc in Electronics Engineering from the same institution in 2009 which is now known as Universiti Teknikal Malaysia Melaka. He received his $\mathrm{PhD}$ in Electrical Engineering and Electronic Research from Brunel University London, United Kingdom in 2017. His research interest is in wireless sensor network using IEEE 802.11 and IEEE 802.15.4 standards. He has a particular interest in a wireless sensor network, IoT system, system integration and consumer electronics system with a good track record of successful industrial collaboration for the past 10 years.

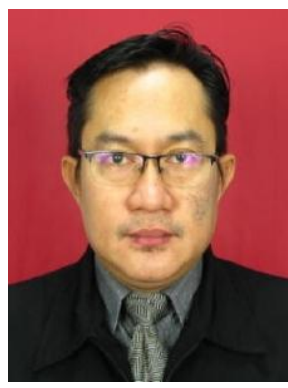

Dr Ismail @ Ismail Yusuf Panessai received the diploma in Telecommunication Engineering from the Polytechnic of Universitas Hasanuddin Indonesia, bachelor in manufacturing (robotic, automation) UJ Indonesia, master science from Department of Artificial Intelligence in the Technical University UTeM Malaysia, and PhD from Department of Artificial Intelligence in University of Malaya. His main research interests include Internet of Things, Artificial Intelligence in education; and Artificial Intelligence (Evolutionary Algorithm, Meta-heuristic, Expert System, and Fuzzy Logic System) and their application in decision making, operation research, vehicle routing problem (logistics/transportation) and control systems. ismailyusuf.com

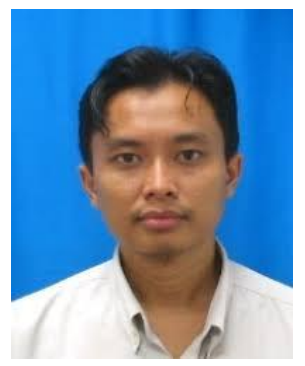

Ir. Ridza Azri Ramlee earned his bachelor's degree in Universiti Teknologi MARA (UiTM)

Shah Alam in 2000, in electrical engineering (electronics industry). In 2006, he was awarded a master's degree in telecommunications engineering and information at the same university. He studied on image processing in $\mathrm{PhD}$, at Universiti Putra Malaysia (UPM). He is a senior lecturer at the Technical University of Melaka since 2008 until now. He had industrial experience as an electrical and biomedical engineer at UKM Hospital from 2001 to 2006. He obtained the Professional Engineer with Practising Certificate (PEPC) status from the Board of Engineer (BEM). This due to the experience gained in industrial work in the Electronic field.

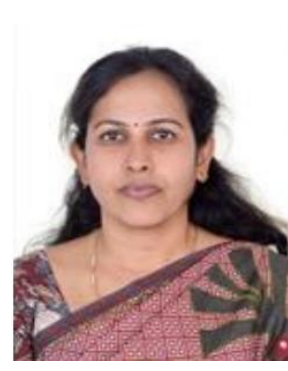

Dr. Sujatha is an Associate Professor in the Department of Embedded Technology, IoT and Sensors Division, School of Electronics Engineering, VIT University, Vellore Campus. Received Ph.D from Anna University, Chennai in the field of Information security . Having 20 years of teaching and research experience in reputed Institutions. Current Research work includes Information security for Big data in Internet of Things, Machine Learning and Deep learning Algorithms for predictive modelling. Focus is on investigating how information security algorithms can be applied to improve the security of IoT data in the cloud environment. Received IETE Best ISF award in the year 2010 and 2011. Lifetime member of IETE professional society. Published more than 15 research articles in peer reviewed academic international journals and conferences.

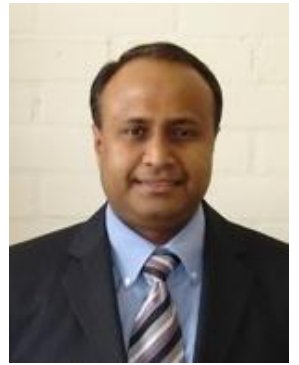

Dr. Rajagopal Nilavalan obtained the B.Sc. Eng. in Electrical \& Electronic Engineering (Firs Class) from University of Peradeniya, SriLanka in 1995 and his $\mathrm{PhD}$ in Near-field microwave imaging from University of Bristol, UK in 2001. From 1999 to 2005 he was a researcher at Centre for Communications Research (CCR), Bristol University working in the field of Radio Frequency Engineering. He was member of the European commission, Network of Excellence on Antennas from 2002 - 2005. He joined Brunel lecturer in wireless communications.
University London in September 2005 as a 